\title{
ÍNDICES DE TRAUMA EM PACIENTES SUBMETIDOS À LAPAROTOMIA
}

\section{TRAUMA SCORING IN PATIENTS SUBMITTED TO LAPAROTOMY}

\author{
Gustavo Pereira Fraga, TCBC-SP'후 Mario Mantovani, TCBC-SP²; Luís Alberto Magna ${ }^{3}$
}

\begin{abstract}
RESUMO: Objetivo: O trauma é um problema de saúde pública de enormes proporções. Constitui-se na principal causa de óbitos na população jovem. O Major Trauma Outcome Study (MTOS) é um estudo descritivo e retrospectivo da gravidade das lesões e evolução dos pacientes, considerado como o maior arquivo contemporâneo de informações descritivas de traumatizados. O objetivo do presente estudo é comparar o cálculo retrospectivo do New Injury Severity Score (NISS) com o Injury Severity Score (ISS) já calculado prospectivamente, utilizando o Trauma and Injury Severity Score (TRISS) e uma simples modificação deste índice, denominado de NTRISS (New Trauma and Injury Severity Score), e também comparar esta população submetida à laparotomia com os pacientes do MTOS. Método: Foram estudados 1.380 pacientes adultos traumatizados e submetidos à laparotomia na Disciplina de Cirurgia do Trauma da Unicamp, em Campinas, durante um período de oito anos. Os dados avaliados foram: demográficos, causa do trauma (fechado ou penetrante, ferimento por projétil de arma de fogo ou arma branca), estado fisiológico na admissão (RTS), diagnóstico anatômico de lesões (ATI, ISS e NISS), probabilidade de sobrevida utilizando o TRISS e o NTRISS, e a evolução do paciente (sobrevivência ou óbito). Foram utilizadas as estatísticas $\mathrm{Z}$ e W, inicialmente descritas por Flora, a fim de comparar a predição de óbitos ou sobreviventes com o estudo controle (MTOS).Resultados: A maioria dos pacientes (88,3\%) era do sexo masculino e jovem (média de idade de 30,4 anos). O ferimento por projétil de arma de fogo foi o mecanismo de trauma mais freqüente, com 641 casos $(46,4 \%)$. Quatrocentos e trinta pacientes $(31,2 \%)$ sofreram trauma fechado. As médias do ATI, ISS e NISS foram, respectivamente, de 12,3, 17,6 e 22,1. A taxa global de mortalidade foi de $16,8 \%$ e os pacientes com trauma contuso tiveram a maior mortalidade $(29,3 \%)$. O NISS identificou melhor os sobreviventes e óbitos se comparado ao ISS, obtendo-se uma maior especificidade com o NTRISS. Foi observado um número significativamente menor de sobreviventes do que o esperado pelo estudo basal, com Z -16,24 com o TRISS e Z -9,40 se aplicado o NTRISS. Variações no valor da estatística W para cada paciente mostraram uma diferença no número de óbitos equivalente a 7,89 mais casos de óbito do que o esperado pelo MTOS, por 100 pacientes tratados, ao se empregar o TRISS, enquanto que estes valores foram reduzidos para 5,14 utilizando-se o NTRISS. Conclusões: Os métodos utilizados para cálculo da probabilidade de sobrevivência apresentaram limitações, particularmente nesta população com predomínio dos traumas penetrantes. O NISS, com o seu derivado NTRISS, foi o escore que obteve uma melhor predição de sobrevivência se comparado com o ISS. Os resultados obtidos com o TRISS e NTRISS foram estatisticamente piores do que os do MTOS, porém este processo de monitorização destes pacientes traumatizados tem sido importante para assegurar uma condição continuada de controle de qualidade (Rev. Col. Bras. Cir. 2004; 31(5): 299-306) - ISSN 0100-6991.
\end{abstract}

Descritores: Trauma; Índices de trauma; Laparotomia; TRISS.

\section{INTRODUÇÃO}

O Brasil é um país com 170 milhões de habitantes e anualmente cerca de 130.000 pessoas morrem vítimas de causas externas. Neste país não existe ainda um sistema organizado de atendimento ao traumatizado, e no sentido de melhorar a assistência a estes pacientes se faz necessário um entendimento detalhado das causas, tratamento e evolução das vítimas destas lesões. E dentro deste programa de controle de qualidade contínuo e efetivo é fundamental a monitorização dos eventos relacionados aos cuidados e evolução do traumatizado, o que é feito através dos registros de trauma, que devem coletar dados referentes aos traumatismos, classificá-los, definir a gravidade e agrupar as informações para análises individuais e coletivas ${ }^{1}$.

Um dos problemas na abordagem do paciente traumatizado é que grupos de pessoas lesadas diferem entre si quanto à natureza e gravidade das lesões ${ }^{2}$. Esta heterogeneidade e a dificuldade em ajustar tais variações têm estimulado o estu- do científico envolvendo as pessoas vítimas de traumatismo. Por isto, foram criados os Índices ou Escores de Trauma, que são valores matemáticos ou estatísticos, quantificados por escores numéricos, que variam de acordo com a gravidade das lesões resultantes do traumatismo ${ }^{3-6}$. Alguns índices são muito específicos, porém outros são de aceitação universal, sendo periodicamente revistos e atualizados a fim de se tornarem mais precisos. O TRISS (Trauma Injury Severity Score) vem sendo um índice adotado, e sob a coordenação do Comitê de Trauma do Colégio Americano de Cirurgiões ("American College of Surgeons Committee on Trauma") foi desenvolvido um estudo para avaliar os resultados de pacientes traumatizados, denominado "Major Trauma Outcome Study" ou MTOS ${ }^{7,8}$. O objetivo inicial deste estudo era desenvolver normas para o tratamento dos traumatizados que pudessem ser adotadas nos hospitais a fim de garantir uma qualidade de atendimento nos sistemas de urgência.

Entre os índices anatômicos descritos na literatura, existem controvérsias em relação à precisão destes ao

1. Professor Assistente Doutor da Disciplina de Cirurgia do Trauma da FCM-Unicamp e do Departamento de Cirurgia do Hospital Municipal "Dr. Mário Gatti”, Campinas-SP

2. Professor Titular e Chefe da Disciplina de Cirurgia do Trauma da FCM-Unicamp

3. Professor Titular do Departamento de Genética Médica da FCM-Unicamp

Recebido em 21/10/2003

Aceito para publicação em 03/06/2004

Trabalho realizado na Disciplina de Cirurgia do Trauma do Departamento de Cirurgia da Faculdade de Ciências Médicas (FCM) da Universidade Estadual de Campinas (Unicamp); Tese de Doutorado do Curso de Pós-Graduação em Cirurgia da FCM - Unicamp. 
quantificar a gravidade das lesões em pacientes com trauma abdominal. Classicamente é utilizado o Índice de Gravidade da Lesão ("Injury Severity Score" ou ISS), que é um escore anatômico que permite classificar e pontuar lesões em diferentes segmentos corpóreos, incluindo o abdome ${ }^{2}$. Inclusive o ISS é o índice utilizado para cálculo do TRISS ${ }^{7}$. Posteriormente foram criados o Índice de Trauma Abdominal Penetrante ("Penetrating Abdominal Trauma Index" ou PATI) e o Índice de Trauma Abdominal ("Abdominal Trauma Index" ou ATI), que avaliam lesões intra-abdominais decorrentes de trauma e identificam os pacientes com alto risco de desenvolverem complicações pós-operatórias ${ }^{9,10}$. E mais recentemente foi descrito o Novo Índice de Gravidade da Lesão ("New Injury Severity Score" ou NISS), a fim de suprimir algumas falhas apresentadas pelo ISS ${ }^{11}$.

O presente estudo descreve um novo índice misto, denominado NTRISS, ainda não descrito na literatura, que para ser calculado utiliza os mesmos critérios do TRISS descritos por Boyd et al. ${ }^{7}$, porém adotando o NISS como índice anatômico ao invés do ISS.

Nos últimos anos a literatura está farta em artigos criticando a acurácia de tais índices, e novos índices de trauma têm sido propostos, o que tem gerado muita polêmica, abrindo perspectivas para novas discussões e adoção de um índice de sobrevida ideal ${ }^{12}$.

Isto posto, os objetivos do presente trabalho são:

a) analisar aspectos epidemiológicos (idade, sexo e mecanismo de trauma) relativos a pacientes submetidos à laparotomia por trauma abdominal;

b) avaliar comparativamente qual o melhor índice misto (TRISS ou NTRISS) para a predição de probabilidade de sobrevida na população estudada, utilizando os respectivos índices anatômicos, ISS e NISS;

c) comparar a população em estudo com os resultados do "Major Trauma Outcome Study" (MTOS).

\section{MÉTODO}

O trabalho foi desenvolvido na Disciplina de Cirurgia do Trauma da Unicamp, que é a responsável pelo atendimento das urgências cirúrgicas traumáticas e não-traumáticas no Hospital das Clínicas da Unicamp, situado na cidade de Campinas, cuja área metropolitana engloba uma população de 2,4 milhões de habitantes. O atendimento inicial ao traumatizado segue os protocolos do serviço que se apóiam no Suporte Avançado de Vida no Trauma - ATLS ${ }^{\circledR}{ }^{13}$. Todas as cirurgias são rigorosamente registradas em protocolos estabelecidos, em programa Epi-Info, versão 6.04, sendo rotineiro o cálculo e anotação dos índices de trauma.

Foram avaliados os prontuários e registros apenas dos pacientes vítimas de trauma operados no período de $1^{\circ} \mathrm{de}$ janeiro de 1994 a 31 de dezembro de 2001. Neste período de oito anos foram realizadas 8.651 cirurgias de urgência, incluindo as reoperações. Destas cirurgias, $1.769(20,4 \%)$ se referiam a procedimentos cirúrgicos em casos novos de traumatismo. Todos os pacientes vítimas de trauma no período estabelecido, submetidos à laparotomia, foram incluídos no estudo, totalizando 1.380 casos. Isto incluiu 1.369 pacientes, uma vez que 11 deles $(0,8 \%)$ foram vítimas de traumas repetidos, em épocas distintas, sendo considerados como casos diferentes.

Em relação ao mecanismo de trauma, os pacientes foram subdivididos em três grupos, a saber: trauma abdominal fechado (TF), ferimento por projétil de arma de fogo (FPAF) e ferimento por arma branca (FAB). Nos poucos casos em que houve a associação destes mecanismos de trauma, o paciente foi incluído no grupo cujo mecanismo resultou na lesão mais grave. O estudo pode ser considerado como prospectivo, porém alguns dados, em especial o NISS, foram calculados mediante a revisão do prontuário, retrospectivamente.

Os dados epidemiológicos analisados foram: gênero, idade, mecanismo de trauma, índice fisiológico (RTS), índices anatômicos (ISS, NISS, ATI), gravidade das lesões anatômicas abdominais (OIS), laparotomias não-terapêuticas, cirurgias associadas, morbidade, tempo de permanência hospitalar, índices mistos (TRISS e NTRISS) e mortalidade.

O RTS foi calculado com os dados vitais (GCS, PAS e FR) da admissão hospitalar, independente de o paciente ter recebido atendimento ou terapêutica pré-hospitalar. O ISS foi calculado usando-se a AIS, segundo a revisão de $1990^{2,14}$. A lesão mais grave da região abdominal foi identificada e quantificada durante a laparotomia. Nos demais segmentos corpóreos, foram utilizados para definição da gravidade das lesões o exame clínico, exames radiográficos, tomografia computadorizada ou outros métodos propedêuticos complementares, além de cirurgias associadas. O NISS foi calculado como descrito por Osler et al., também usando a AIS de 1990" ${ }^{11}$. O ATI calculado neste estudo usou como referência os fatores de risco descritos por Borlase et al. ${ }^{10}$. A probabilidade de sobrevida foi estimada utilizando-se a tabela TRISSCAN ${ }^{7}$. Porém, foi testada uma outra variação de índice anatômico e de referência, que resultou em diferentes probabilidades, sendo:

- TRISS, obtido com o uso do ISS;

- NTRISS, obtido com o uso do NISS.

Independente do índice anatômico utilizado, ISS ou NISS, a probabilidade de sobrevida foi obtida com a associação do RTS e faixa etária convencionalmente utilizados (= 54 anos ou $>54$ anos).

Por convenção, os óbitos consequientes a trauma com TRISS/NTRISS menor que o valor 0.50 foram definidos como esperados ou inevitáveis, enquanto que mortes com TRISS/NTRISS com valores maiores ou iguais a 0.50 constituíram evoluções não esperadas.

A taxa de mortalidade do presente estudo foi comparada com a estimada pelo MTOS usando-se os métodos TRISS e NTRISS, por meio das metodologias denominadas estatística $\mathrm{Z}$ e estatística $\mathrm{W}^{7,14}$.

A sensibilidade dos índices mistos avaliados foi calculada pela probabilidade condicional de sobrevivência no grupo de sobreviventes e a especificidade pela probabilidade condicional de morte no grupo de óbitos. A taxa de pacientes com erro de classificação foi obtida pela soma de falsos positivos e negativos, e divisão pelo número total de pacientes.

Conforme a natureza das variáveis estudadas, utilizou-se para análise dos resultados o teste do Qui-quadrado, para comparação de tabelas de contingência. O nível crítico 
para a rejeição da hipótese da nulidade, a partir do qual a diferença foi considerada como estatisticamente significativa, foi de $5 \%(\mathrm{a} \leq 0,05)$, assinalando-se com um asterisco os valores estatisticamente significativos.

\section{RESULTADOS}

Nos 1.380 casos estudados, a distribuição conforme o gênero foi: masculino ( 1.218 casos - 88,3\%) e feminino (162 casos $-11,7 \%)$. Os 11 pacientes $(0,8 \%)$ vítimas de traumatismos penetrantes em períodos diferentes, que constaram duas vezes da casuística, eram do sexo masculino. Em relação à faixa etária, houve uma variação dos 13 aos 79 anos de idade, com uma média da população estudada de 30,4 anos. Observou-se que 76 pacientes $(5,5 \%)$ tinham idade maior que 54 anos.

Quanto ao mecanismo do trauma, houve predomínio dos traumatismos penetrantes (950 casos - 68,8\%), que foram divididos em FPAF e FAB. A Tabela 1 mostra a freqüência dos mecanismos de trauma.

A maioria dos pacientes (1.011 casos - 73,3\%) foi admitida com RTS de 7,84, com média de 7,19. De acordo com o mecanismo de trauma, a média do RTS foi de 6,62 nos traumas contusos; 7,41 nos FPAF; e de 7,52 nos FAB.

Em relação aos achados na laparotomia exploradora, o fígado (31,3\%), jejuno/íleo (24,8\%) e cólon (22\%) foram os órgãos mais acometidos. O ATI foi maior que 25 em 183 casos $(13,3 \%)$, conforme ilustrado no Gráfico 1 . Houve uma variação deste índice de 0 a 69, com média de 12,3. Em relação aos índices anatômicos ISS e NISS, a maior pontuação destes escores foi de 66. A média do ISS foi de 17,6 e do NISS de 22,1. Como pode ser observado, 549 pacientes $(39,8 \%)$ tinham NISS $>25$, enquanto que apenas 294 casos $(21,3 \%)$ foram classificados com ISS > 25. A pontuação do ISS e do NISS foi a mesma em 477 pacientes $(34,6 \%)$, enquanto que em 903 casos $(65,4 \%)$ o NISS era maior que o ISS.

Os valores dos índices anatômicos variaram bastante conforme o mecanismo de trauma. A Tabela 2 mostra as médias dos diferentes índices de acordo com o tipo de traumatismo.

Observou-se que nos pacientes com trauma contuso as pontuações foram mais altas em índices que avaliaram todos os segmentos corpóreos (ISS e NISS), uma vez que estes traumatizados freqüentemente têm lesões associadas extraabdominais. Já nos traumas penetrantes, a média do ATI se aproximou da média do ISS, isto em decorrência dos FPAF, uma vez que estes pacientes freqüentemente têm múltiplas lesões associadas no segmento abdominal. Como o NISS é um índice anatômico que pontua estas lesões, mesmo se localizadas apenas no segmento abdominal, elevando as pontuações ao quadrado, observaram-se valores maiores deste índice nos pacientes vítimas de FPAF (Tabela 2).

A mortalidade foi de 16,8\% (232 casos), conforme ilustrado na Tabela 3 . Ao se analisar a mortalidade secundária aos diferentes mecanismos de trauma, foi verificada maior taxa de óbitos nos traumas fechados, o que foi estatisticamente significativo comparado com os traumas penetrantes $(p<0,001 *)$. Dentre os traumas penetrantes, também foi observada uma diferença com significância estatística $\left(p<0,05^{*}\right)$, pelo teste do Qui-quadrado, ao se comparar os FPAF e FAB, com uma menor mortalidade nestes últimos, conforme observado na Tabela 3 .

A incidência de óbitos conforme os índices anatômicos (ATI, ISS e NISS) foi avaliada dividindo-se os pacientes em dois subgrupos: escore menor ou igual a 25 ou escore maior que 25. Comparando-se estes subgrupos utilizando os diferentes índices, observou-se uma mortalidade de, respectivamente: $13,5 \%$ e $38,3 \%$, com ATI $\leq 25$ e ATI > 25; 8,8\% e 46,3\%, ao analisar o ISS; e $4,8 \%$ versus $35 \%$ de mortalidade nos pacientes com NISS > 25. Aplicando o teste do Qui-quadrado, foi verificada uma diferença estatisticamente significativa entre os subgrupos ( $p<0,0001 *)$ com os três índices testados.

Quanto à probabilidade de sobrevida, a Tabela 4 mostra a sensibilidade, especificidade, falsa positividade, falsa negatividade e erros de classificação dos diferentes índices testados: TRISS e NTRISS. O NTRISS foi o índice que mostrou um melhor desempenho para inferir a probabilidade de sobrevida na população estudada.

A aplicação das estatísticas $\mathrm{Z}$ e W aos dois índices mistos avaliados mostrou resultados inferiores aos obtidos no MTOS (Tabela 5). Isto porque ao se aplicar a estatística Z para sobreviventes os valores foram negativos, tanto do TRISS ($16,24)$ como do NTRISS $(-9,40)$, e fora da faixa que mostraria igualdade estatisticamente significativa entre a população do estudo e o MTOS, ou seja, até -1,96. Na análise da estatística Z, para óbitos, observou-se um valor derivado do TRISS de +16,24 e do

Tabela 1 - Número de casos conforme o mecanismo de trauma.

\begin{tabular}{lcc}
\hline $\begin{array}{l}\text { MECANISMO } \\
\text { DETRAUMA }\end{array}$ & $\begin{array}{c}\text { NÚMERO } \\
\text { DE CASOS }\end{array}$ & PORCENTAGEM \\
\hline Fechado & 430 & 31,2 \\
FPAF & 641 & 46,4 \\
FAB & 309 & 22,4 \\
Total & $\mathbf{1 . 3 8 0}$ & $\mathbf{1 0 0 , 0}$ \\
\hline
\end{tabular}

Tabela 2 - Variação dos índices anatômicos (médias) conforme o mecanismo de trauma.

\begin{tabular}{lccc}
\hline MECANISMO & \multicolumn{3}{c}{ ÍNDICE (média) } \\
DETRAUMA & ATI & ISS & NISS \\
\hline Fechado & 10,6 & 23,5 & 25,6 \\
Penetrante & 13,0 & 14,9 & 20,5 \\
· FPAF & 15,4 & 16,9 & 23,5 \\
· FAB & 8,2 & 10,7 & 14,2 \\
\hline
\end{tabular}

Tabela 3 - Número de casos e mortalidade conforme o mecanismo de trauma.

\begin{tabular}{lccc}
\hline \multicolumn{2}{c}{ MECANISMODETRAUMA } & \multicolumn{2}{c}{ MORTALIDADE } \\
& $\mathbf{n}$ & $\mathbf{n}$ & $\mathbf{\%}$ \\
\hline Fechado & 430 & 126 & 29,3 \\
Penetrante & 950 & 106 & 11,2 \\
$\quad$ FPAF & 641 & 84 & 13,1 \\
FAB & 309 & 22 & 7,1 \\
Total & 1.380 & 232 & 16,8 \\
\hline
\end{tabular}




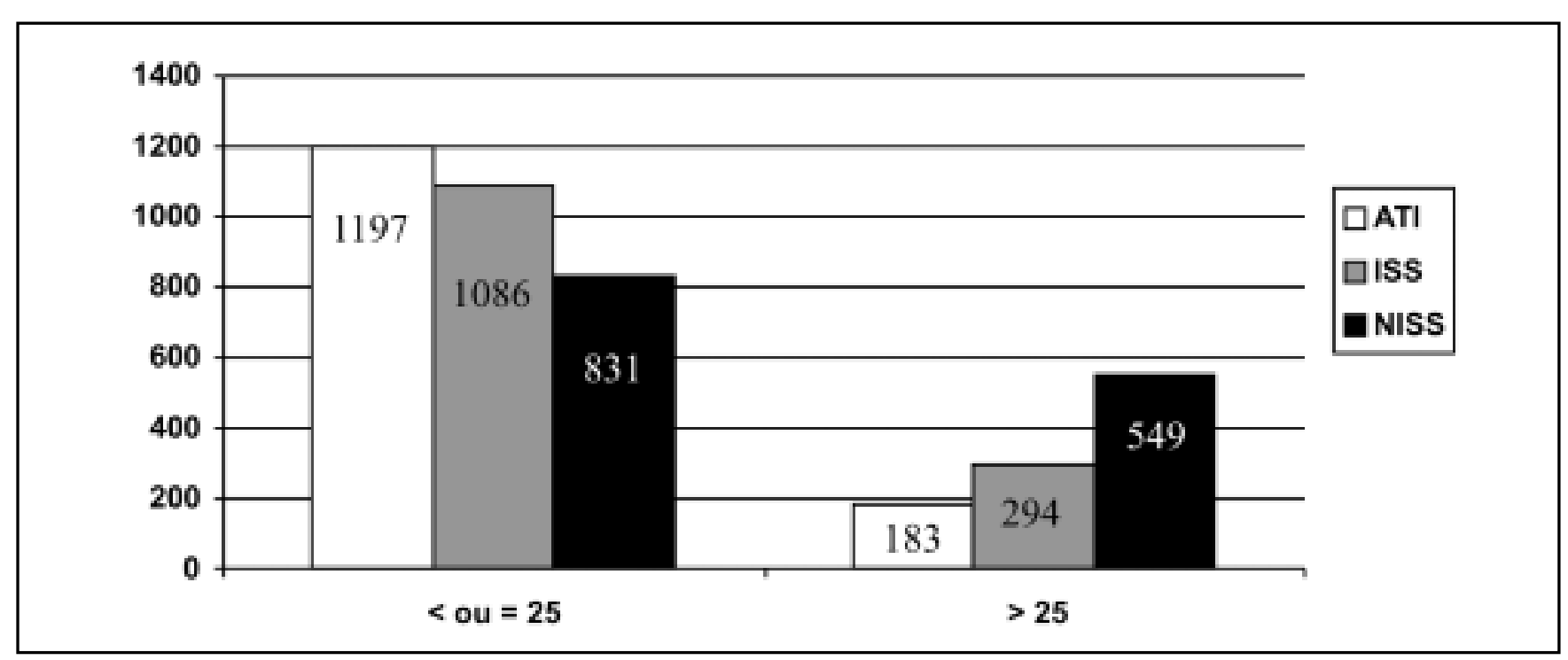

Gráfico 1 - Número de casos conforme a pontuação de escores anatômicos.

NTRISS de $+9,40$, porém o ideal seria que estes valores fossem negativos. Os valores obtidos com a estatística W mostraram que a sobrevida dos pacientes estimada no presente estudo esteve abaixo da descrita pelo MTOS (TRISS: -7,89 e NTRISS: -5,14), e que a incidência de óbitos foi maior. Houve um melhor desempenho do método NTRISS, conforme observado na Tabela 5.

\section{DISCUSSÃO}

No presente estudo houve predomínio dos traumatismos em indivíduos do sexo masculino (88,3\% dos casos) e na faixa etária de adulto jovem, o que também é observado na literatura ${ }^{8,15,16}$.

Tabela 4 - Propriedades dos diferentes índices de probabilidade de sobrevida testados, com resultados expressos em porcentagem.

\begin{tabular}{lcc}
\hline PROPRIEDADES & \multicolumn{2}{c}{ ÍNDICES TESTADOS } \\
DOS ÍNDICES & TRISS & NTRISS \\
\hline Sensibilidade & 98,5 & 97,4 \\
Especificidade & 37,9 & 45,7 \\
Falsa positividade & 11,3 & 10,1 \\
Falsa negatividade & 16,2 & 22,0 \\
Erro de classificação & 11,7 & 11,3 \\
Média & 91,1 & 88,4 \\
\hline
\end{tabular}

Tabela 5 - Resultados obtidos com aplicação das fórmulas das estatísticas Z e W na população estudo, utilizando-se os índices mistos testados.

\begin{tabular}{lcc}
\hline ESTATISTICA & \multicolumn{2}{c}{ ÍNDICESTESTADOS } \\
& TRISS & NTRISS \\
\hline Z para sobreviventes & $-16,24$ & $-9,40$ \\
Z para óbitos & $+16,24$ & $+9,40$ \\
W para sobreviventes & $-7,89$ & $-5,14$ \\
W para óbitos & $+7,89$ & $+5,14$ \\
\hline
\end{tabular}

Em relação ao mecanismo de trauma, o que tem sido observado neste serviço é um aumento na incidência de traumas penetrantes, principalmente os causados por projéteis de arma de fogo, o que é reflexo da violência que se dissemina pela região. Obviamente, cada região tem um próprio perfil em relação aos mecanismos e tipos de trauma, e isto também varia ao se comparar hospitais diferentes ${ }^{17}$. A mortalidade e a aplicação de determinados índices de trauma variam bastante, conforme o local estudado, sendo necessária muita atenção na avaliação dos resultados, para se evitar conclusões precipitadas ou errôneas. Em serviços em que há predomínio de traumas penetrantes, geralmente ocorrem muitas limitações no uso do ISS e do TRISS ${ }^{17-19}$.

Cayten et $a l .{ }^{17}$ notaram limitação do TRISS ao fazerem comparação entre diferentes hospitais. A taxa de mortalidade num serviço com elevada proporção de FPAF seria nitidamente maior que a taxa de óbitos de outro serviço em que houvesse predomínio dos traumas por arma branca. As diferenças de probabilidade de sobrevida utilizando o TRISS não seriam adequadas, motivo pelo qual os autores sugeriram o desenvolvimento de coeficientes do TRISS separando os dois principais tipos de trauma penetrante. No presente estudo, a variação nas taxas de mortalidade entre FPAF e FAB também foi estatisticamente significativa (Tabela 3), sendo de, respectivamente, $13,1 \%$ e $7,1 \%$.

Outra limitação do método TRISS é a ineficiência ao avaliar pacientes com múltiplas lesões graves no mesmo segmento corpóreo, uma vez que estas não são somadas ao calcular o ISS, o que geralmente resulta num ISS de $25^{17,19}$. Isto é bem exemplificado nos pacientes com FPAF no abdome, o que geralmente resulta em várias lesões em diferentes órgãos e vísceras. Em estudo publicado por Cornwell III et al.$^{19}$, foram avaliados 848 pacientes com FPAF no abdome atendidos num grande centro de trauma e foram revistos os prontuários de 39 pacientes ( $36 \%$ dos casos de óbito) considerados óbitos inesperados, ou seja, com probabilidade de sobrevida estimada maior que $50 \%$ (TRISS > 0.50). Estes pacientes tinham média do PATI de 40,3 e a probabilidade de sobrevida média estima- 
da para estes 39 pacientes mortos foi de $89 \%$. A revisão crítica dos casos estimou que o óbito era não evitável em 38 pacientes $(97 \%)$ e potencialmente evitável em 1 caso (3\%). Os autores concluíram que o TRISS é um método muito limitado para ser usado em pacientes com FPAF no abdome. Conclusões semelhantes foram obtidas por Ordoñez et al. ${ }^{18}$, que num estudo em 621 pacientes traumatizados atendidos num serviço de Cali, na Colômbia, tiveram uma incidência de 90,1\% dos casos secundários à trauma penetrante. Afirmaram que naquele país não era aconselhável o uso do ISS como guia, uma vez que a maioria dos casos tem lesões em um ou dois sistemas (tórax e abdome), ao contrário do que é observado em países onde predominam os traumatismos fechados.

No presente estudo foi feita uma comparação entre os três índices anatômicos: ATI, ISS e NISS. O ATI avalia apenas lesões abdominais, sendo um bom índice preditor de complicações em pacientes submetidos à laparotomia. Nesta população, os valores obtidos com o ATI foram os menores entre os índices anatômicos. Os valores do ATI foram menores do que do ISS e NISS principalmente nos traumas contusos, além de apresentarem médias menores também em vítimas de FPAF e FAB (Tabela 2). A vantagem em se utilizar o ATI ao invés do ISS baseia-se no fato de que o ATI considera todas as lesões abdominais, ao contrário do ISS, em que somente a lesão mais grave de cada segmento corpóreo é levada em consideração. Em contrapartida, lesões em outros segmentos, que podem contribuir significativamente para aumentar as taxas de morbidade, não são consideradas no $\mathrm{ATI}^{15}$.

A fim de eliminar deficiências do ISS é que Osler et $a l .{ }^{11}$, em 1997, propuseram a utilização do NISS. Na descrição deste índice, os autores utilizaram registros de trauma de dois centros, e o cálculo deste índice foi feito de modo retrospectivo, assim como no presente estudo. Em $60 \%$ dos casos os valores do NISS foram maiores do que os do ISS ${ }^{11}$. A predição de mortalidade pelo NISS foi baseada exclusivamente neste índice anatômico, e os autores concluíram que o NISS é um índice de fácil aplicação e com melhor acurácia do que o ISS, recomendando o seu uso rotineiro.

No presente trabalho, o NISS foi o índice anatômico que apresentou as maiores pontuações. Se comparado com o ISS, 477 pacientes $(34,6 \%)$ tinham a mesma pontuação, enquanto que nos demais 903 casos $(65,4 \%)$ o NISS era maior que o ISS. Na população estudada, $39,8 \%$ dos pacientes tinham NISS > 25, enquanto que apenas $21,3 \%$ dos traumatizados apresentaram ISS > 25 (Gráfico 1). De acordo com o mecanismo de trauma, as médias do NISS foram superiores às do ATI e ISS, principalmente nos traumas penetrantes (Tabela 2). E como há um predomínio deste mecanismo de trauma nos pacientes atendidos neste serviço, o NISS despontou como o índice anatômico com melhor desempenho nesta população de traumatizados. A incidência de complicações e óbito em pacientes com NISS > 25 foi estaticamente superior $(p<0,0001 *)$ do que naqueles com NISS $\leq 25$.

Em estudo realizado na Canadá, Brenneman et al..$^{20}$ avaliaram 2.328 pacientes vítimas de trauma fechado, e compararam os índices ISS e NISS. Em 68\% dos casos a pontuação do NISS foi maior do que a do ISS, sendo as médias destes, respectivamente, 25 e 33. Os pacientes com discrepância entre os índi- ces tiveram maior mortalidade do que aqueles em que o NISS era igual ao ISS, sendo esta diferença estaticamente significativa. Os autores concluíram que o NISS tem uma melhor acurácia na predição de mortalidade nestes traumatizados ${ }^{20}$. Resultados semelhantes foram obtidos em outros estudos ${ }^{21-23}$.

Grisoni et al. ${ }^{24}$ compararam a eficácia do ISS e do NISS na predição de mortalidade em crianças traumatizadas. Avaliando 9.151 pacientes com idade menor que 18 anos, sendo mais de $90 \%$ vítimas de trauma contuso, os autores não encontraram diferenças significativas entre estes índices anatômicos. Husum e Strada ${ }^{25}$ compararam o ISS e o NISS em traumas penetrantes, e os resultados para predição de complicações e mortalidade por estes índices foram semelhantes, porém o estudo foi realizado numa população de baixo risco, com poucos casos de trauma grave. Os autores concluíram que novos estudos se fazem necessários antes de se adotar o NISS como "padrão ouro" entre os índices anatômicos.

No presente trabalho, a mortalidade foi de 16,8\% (232 casos). Trata-se de uma população com características próprias, em que todos os pacientes foram submetidos à laparotomia por trauma abdominal, e 79\% destes tinham lesão de alguma víscera ou órgão que exigiu terapêutica cirúrgica específica.

A avaliação da probabilidade de sobrevida na presente casuística por intermédio do TRISS e do NTRISS mostrou que os dois métodos têm elevada sensibilidade, evidenciando que ambos são excelentes para prever sobrevivência ou morte de um traumatizado (Tabela 4). Já a especificidade ao se aplicar o TRISS e o NTRISS foi baixa. Esta baixa especificidade foi decorrente da evolução inesperada, ou seja, óbito, em traumatizados com probabilidade de sobrevida maior ou igual a $50 \%$, considerados como "mortes não esperadas". O NTRISS se mostrou mais eficiente do que o TRISS na determinação da probabilidade de morte entre as vítimas fatais, sendo os valores, respectivamente, $45,7 \%$ e 37,9\%. A taxa de pacientes com erro de classificação foi muito próxima nos índices testados (11,7\% com o TRISS e 11,3\% com o NTRISS).

No presente estudo, as análises de probabilidade de sobrevida obtidas com o TRISS e NTRISS foram resultantes de seus índices anatômicos, respectivamente, o ISS e o NISS. Os outros parâmetros utilizados para este cálculo, ou seja, o RTS, a idade do paciente ( $\leq 54$ anos ou $>54$ anos) e o mecanismo do trauma (fechado ou penetrante), foram os mesmos. Portanto, a mudança da probabilidade de sobrevida de um determinado paciente, de provável sobrevivência para provável óbito, foi exclusivamente devido a uma maior pontuação do índice anatômico. Como já exposto sobre a maior eficácia do NISS nos traumas penetrantes, este fato explica a ligeira superioridade do NTRISS em relação ao TRISS.

Na literatura, a sensibilidade ao se aplicar o TRISS como preditor de probabilidade de sobrevida varia de $58 \%$ a $100 \%$, enquanto que a especificidade varia de $35 \%$ a $99 \%$. As limitações do TRISS não impedem o seu uso, pelo contrário, exigem uma análise crítica dos resultados referentes à população avaliada.

Os resultados do MTOS são considerados ainda como o padrão de tratamento do traumatizado. Um dos objetivos do presente trabalho foi comparar a população em estudo com a do MTOS. Para isto, foram utilizadas as estatísticas Ze W. É impor- 
Tabela 6 - Aspectos epidemiológicos e gravidade das lesões (índices de trauma) da população do estudo e do MTOS.

\begin{tabular}{lcc}
\hline VARIÁVEL & ESTUDO & MTOS \\
\hline Número de casos & 1.380 & 80.544 \\
Número de instituições & 1 & 139 \\
Período do estudo & $1994-2001$ & $1982-1987$ \\
Características da população & Traumatizados submetidos à laparotomia & Todas as admissões por trauma \\
Casos excluídos & $0,07 \%$ & $11,3 \%$ \\
Faixa etária (média) & 30,4 anos & 33 anos \\
$<15$ anos & - & $10,8 \%$ \\
Mecanismo de trauma & & \\
- fechado & $31,2 \%$ & $78,9 \%$ \\
- penetrante & $68,8 \%$ & $21,1 \%$ \\
RTS (média) & 7,19 & 7,1 \\
ISS (média) & 17,6 & 12,8 \\
Mortalidade & $16,8 \%$ & $9 \%$ \\
\hline
\end{tabular}

tante ressaltar que a população do estudo tem características epidemiológicas e de gravidade de lesões diferentes da população basal (MTOS), conforme apresentado na Tabela 6.

Pela estatística Z, para que os valores absolutos obtidos ao se calcular os sobreviventes e óbitos do grupo-estudo fossem estatisticamente semelhantes aos do MTOS, eles deveriam estar entre $-1,96 \mathrm{e}+1,96$. No cálculo dos sobreviventes, $\mathrm{o}$ ideal seria que fossem obtidos valores positivos, porém a análise com o TRISS resultou em -16,24 e com o NTRISS em -9,40. Já no cálculo dos óbitos aconteceu o mesmo, ou seja, estes mesmos valores absolutos, porém em condição inversa se comparado ao esperado como ideal. Isto significa que os resultados obtidos no estudo foram piores do que os observados no MTOS. No próprio estudo do MTOS, ao se realizar a estatística Z e avaliar a sobrevida dos traumatizados, 7,5\% das instituições que atenderam vítimas de trauma contuso e $12,1 \%$ das que trataram de vítimas de trauma penetrante apresentaram resultados piores do que os considerados como satisfatórios ${ }^{8}$.

A estatística $\mathrm{W}$ também foi testada com o TRISS e o NTRISS. Utilizando o TRISS, observou-se uma taxa de 7,89 casos de óbito para cada 100 pacientes a mais do que o previsto pelo MTOS. Pelo cálculo do NTRISS, este valor caiu para 5,14, mostrando novamente uma maior aproximação dos resultados obtidos com o NTRISS daqueles descritos no MTOS. No MTOS, houve instituições que chegaram a apresentar resultados piores do que estes, com valores absolutos de até 9,5 pela estatística $\mathrm{W}^{8}$.

Em trabalho recente, Demetriades et al. ${ }^{16}$ avaliaram a utilidade e as limitações do método em um grande centro de trauma urbano e concluíram que o TRISS apresenta limitações em vários subgrupos de pacientes, principalmente nos que sofreram traumas graves. O TRISS foi avaliado comparandose a sobrevida esperada com a observada. Os autores observaram que nos pacientes de baixo risco o erro foi em torno de $4,3 \%$ dos pacientes estudados. Entretanto, em pacientes que sofreram traumas graves, com ISS maior que 20, este percentual de erro elevou-se nas seguintes proporções: $34 \%$ em pacientes acima de 54 anos, $29 \%$ nos pacientes com lesões por queda e naqueles com lesões em quatro ou mais regiões do corpo, $26,4 \%$ de erro nos pacientes em choque na admissão e $26,1 \%$ em pacientes que apresentaram complicações durante a internação. Desta forma, além de citarem estas limitações, enfatizaram que o método deve ser melhor interpretado nestas situações de gravidade e o seu papel reconsiderado e talvez até abandonado, sendo necessárias outras alternativas.

No momento, o que se pode concluir é que o TRISS não é um método ideal para estimar a probabilidade individual de sobrevida. Tamanha insatisfação é refletida na literatura, onde novos índices são descritos com freqüência e posteriormente acabam no esquecimento, talvez pelo fato de não agradarem por completo. Atualmente outro índice que tem sido difundido é o ICISS, que é o Índice de Gravidade da Lesão baseado no CID-9 ou Código Internacional de Doenças - 9a edição $^{31}$. Os trabalhos preliminares encabeçados pelas equipes de Rutledge e Osler mostraram que as informações anatômicas das lesões derivadas do CID-9 tinham um valor preditivo significativo quando estratificada a gravidade das lesões s1-33 $^{31}$. Alguns estudos avaliaram a eficiência de índices anatômicos e do TRISS, comparando-os com o ICISS, e observaram mais propriedades e melhor qualidade com o uso do ICISS ${ }^{34,35}$.

O presente estudo mostrou uma maior eficácia do índice denominado NTRISS, que utiliza o NISS como escore anatômico, se comparado ao TRISS. Tal ligeira vantagem do NTRISS nesta população, em que predominaram os traumas penetrantes e com lesões anatômicas numericamente mais graves do que as do MTOS, não foi significativa a ponto de utilizar rotineiramente este índice. Na nossa experiência, o TRISS, apesar de suas limitações, ainda é o índice adotado. Com isto, mantém-se o processo de monitorização do atendimento médico, usando padrões definidos, a fim de identificar problemas nas fases do atendimento que determinam impacto negativo no resultado do tratamento. Embora não fosse exatamente o objetivo deste estudo, a auditoria proporcionada pelos resultados apresentados gerou as seguintes considerações: o uso rotineiro dos índices de trauma permitiu a uniformização da linguagem no respectivo serviço, a realização de diversos estudos clínicos, a reavaliação dos tratamentos, a orientação em reuniões multidisciplinares e a criação de um desafio constante para a equipe na busca de melhores resultados. 


\begin{abstract}
Background: Trauma is a public health problem of vast proportions. It is the leading cause of death among young people. The Major Trauma Outcome Study (MTOS) is a retrospective descriptive study of injury severity and outcome, considered the largest database of descriptive contemporary injuries information. The objective of this paper is to compare the retrospective calculation of NISS with prospectively calculated ISS, using TRISS and a simple modification called NTRISS (New Trauma and Injury Severity Score), and to compare this population submitted to laparotomy with MTOS patients. Methods: We studied 1.380 trauma adult patients submitted to laparotomy at Discipline of Trauma Surgery - Unicamp, in Campinas, during a 8-year period. Submitted data included: demographics, cause of injury (blunt or penetrating, gunshot or stab wounds), physiologic status at admission (RTS), anatomic injury diagnoses (ATI, ISS and NISS), survival probability using the TRISS and NTRISS, and patient outcomes (survival or death). Z statistic and W statistic, first described by Flora, were used to compare the predicted number of deaths (or survivors) with the baseline MTOS norm. Results: The majority of these patients was male (88.3\%) with a mean age of 30.4 years. Gunshot wounds were the most frequent (641 cases - 46.4\%) mechanism of trauma. Thirty one per cent had blunt injuries. The median ATI, ISS and NISS values were, respectively, 12.3, 17.6 and 22.1. The overall mortality rate was $16.8 \%$ and the patients with blunt trauma had the highest mortality rate (29.3\%). NISS better separated survivors from nonsurvivors than ISS, with higher specificity of NTRISS. It was observed a significantly less survivors than expected from outcomes norms (Z -16.24 with TRISS and Z -9.40 with NTRISS). Ranges of W values for each patient set demonstrated a difference in the numbers of nonsurvivors of 7.89 more deaths per 100 patients treated than expected from the MTOS using TRISS, while this values were reduced to 5.14 using NTRISS. Conclusions: The methods used for survival probability calculation presented limitations, particularly in this population with predominance of penetrating trauma. The NISS, with its derived NTRISS, was the score that better predicted survival than ISS. The results obtained with TRISS and NTRISS were significantly worse than MTOS, but this process of monitoring trauma patients have been important to ensure continued provision of quality of care.
\end{abstract}

Key words: Trauma; Trauma scoring; Laparotomy; TRISS.

\section{REFERÊNCIAS}

1. Rutledge $\mathrm{R}$ - The goals, development, and use of trauma registries and trauma data sources in decision making in injury. Surg Clin North Am, 1995, 75(2):305-326.

2. Baker SP, O'Neill B, Haddon W, et al. - The injury severity score: a method for describing patients with multiple injuries and evaluating emergency care. J Trauma, 1974, 14(3):187-196.

3. Fontes B - "Índices de Trauma". In Birolini D, Utiyama E, Steinman E - Cirurgia de emergência. São Paulo. Editora Atheneu, 1997, pp.142-147.

4. Birolini D - Trauma: os índices de trauma. Rev Col Bras Cir, 1996, 23(1):4-5.

5. Coimbra RSM, Angle N, Silva LE, et al. - Índices de trauma: o que são e por que devem ser usados? Rev Col Bras Cir, 1997, 24:255-263.

6. Mantovani M, Fraga GP - "Avaliação da gravidade. Escalas de Trauma”. In Freire E - Trauma - a doença dos séculos. $1^{\text {a }}$ Edição. Rio de Janeiro. Editora Atheneu, 2001, pp.403-420.

7. Boyd CR, Tolson MA, Copes WS - Evaluating trauma care: the TRISS method. Trauma Score and the Injury Severity Score. J Trauma, 1987, 27(4):370-378.

8. Champion HR, Copes WS, Sacco WJ, et al. - The Major Trauma Outcome Study: establishing national norms for trauma care. J Trauma, 1990, 30(11):1356-1365.

9. Moore EE, Dunn EL, Moore JB, et al. - Penetrating abdominal trauma index. J Trauma, 1981, 21(6):439-445.

10. Borlase BC, Moore EE, Moore FA - The abdominal trauma index -- a critical reassessment and validation. J Trauma, 1990, 30(11):1340-1344.

11. Osler T, Baker SP, Long W - A modification of the injury severity score that both improves accuracy and simplifies scoring. J Trauma, 1997, 43(6):922-926.

12. Mantovani M, Fraga GP - "Mortalidade e índices de sobrevida no trauma". In Freire E - Trauma - a doença dos séculos. $1^{\mathrm{a}}$ Edição. Rio de Janeiro. Editora Atheneu, 2001, pp. 2873-2884.
13. American College of Surgeons. Committee on Trauma. Advanced Trauma Life Support Program (ATLSÒ). Instructor Manual. Chicago: American College of Surgeons, 1997.

14. Flora JD - A method for comparing survival of burn patients to a standard survival curve. J Trauma, 1978, 18(10):701-705.

15. Ribeiro Júnior MAF, Borghesi RA, Eshkenazy R, et al. - Índice de Trauma Abdominal: aplicabilidade clínica e valor prognóstico. Rev Col Bras Cir, 1996, 23(3):139-142.

16. Demetriades D, Chan LS, Velmahos G, et al. - TRISS methodology in trauma: the need for alternatives. Brit J Surg, 1998, 85(3):379-384.

17. Cayten CG, Stahl WM, Murphy JG, et al. - Limitations of the TRISS method for interhospital comparisons: a multihospital study. J Trauma, 1991, 31(4):471-482.

18. Ordoñez C, Becerra C, Ferrada R - Índice de trauma como predictores de mortalidad y complicaciones. Panam J Trauma, 1990, 2:44-50.

19. Cornwell EE, Velmahos GC, Berne TV, et al. - Lethal abdominal gunshot wounds at a level I trauma center: analysis of TRISS (Revised Trauma Score and Injury Severity Score) fallouts. J Am Coll Surg, 1998, 187(2):123-129.

20. Brenneman FD, Boulanger BR, McLellan BA, et al. - Measuring injury severity: time for a change? J Trauma, 1998, 44(4):580582.

21. Balogh Z, Offner PJ, Moore EE, et al. - NISS predicts postinjury multiple organ failure better than the ISS. J Trauma, 2000, 48(4):624-628.

22. Jamulitrat $S$, Sangkerd $P$, Thongpiyapoom $S$, et al. - A comparison of mortality predictive abilities between NISS and ISS in trauma patients. J Med Assoc Thai, 2001, 84(10):1416-1421.

23. Streng M, Tikka S, Leppaniemi A - Assessing the severity of truncal gunshot wounds: a nation-wide analysis from Finland. Ann Chir Gynaecol, 2001, 90(4):246-251.

24. Grisoni E, Stallion A, Nance ML, et al. - The New Injury Severity Score and the evaluation of pediatric trauma. J Trauma, 2001, 50(6):1106-1110. 
25. Husum H, Strada G - Injury Severity Score versus New Injury Severity Score for penetrating injuries. Prehospital Disaster Med, 2002, 17(1):27-32.

26. Hill DA, Lennox AF, Neil MJ, et al. - Evaluation of TRISS as a means of selecting trauma deaths for clinical peer review. Austr N Z J Surg, 1992, 62(3):204-208.

27. Offner PJ, Jurkovich GJ, Gurney J, et al. - Revision of TRISS for intubated patients. J Trauma, 1992, 32(1):32-35.

28. Corbanese U, Possamai C, Casagrande L, et al. - Evaluation of trauma care: validation of the TRISS method in an Italian ICU. Intensive Care Med, 1996, 22(9):941-946.

29. Garber BG, Hebert PC, Wells G, et al. - Validation of trauma and injury severity score in blunt trauma patients by using a Canadian trauma registry. J Trauma, 1996, 40(5):733-737.

30. Garber BG, Hebert PC, Wells G, et al. - Differential performance of TRISS-like in early and late blunt trauma deaths. J Trauma, 1997, 43(1):1-7.

31. Osler T, Rutledge R, Deis J, et al. - ICISS: an international classification of disease- 9 based injury severity score. J Trauma, 1996, 41(3):380-388.

32. Rutledge R, Fakhry S, Rutherford E, et al. - Comparison of APACHE II, Trauma Score, and Injury Severity Score as predictors of outcome in critically injured trauma patients. Am J Surg, 1993, 166(3):244-247.
33. Rutledge R - Injury severity and probality of survival assessment in trauma patients using a predictive hierarchical network model derived from ICD-9 codes. J Trauma, 1995 , 38(4):590-601.

34. Rutledge R, Osler T, Emery S, et al. - The end of the Injury Severity Score (ISS) and the Trauma and Injury Severity Score (TRISS): ICISS, an International Classification of Diseases, ninth revision-based prediction tool, outperforms both ISS and TRISS as predictors of trauma patient survival, hospital charges, and hospital length of stay. J Trauma, 1998, 44(1):41-49.

35. Meredith JW, Evans G, Kilgo PD, et al. - A comparison of the abilities of nine scoring algorithms in predicting mortality. $\mathrm{J}$ Trauma, 2002, 53(4):621-629.

Endereço para correspondência:

Gustavo Pereira Fraga

Av. Coronel Silva Telles, 211 apto 3

13024-000 Cambuí Campinas-SP

Telefone: (19) 3294-6348 celular: (19) 9772-0829

fragagp@uol.com.br 